UM-P-93/38

KCL-93-7

9306088

May 1993

\title{
The Space of Solutions of Toda Field Theory
}

\author{
G. PAPADOPOULOS \\ Department of Mathematics \\ King's College London \\ London WC2R 2LS \\ and \\ B. SPENCE \\ School of Physics \\ University of Melbourne \\ Parkville 3052 Australia
}

\begin{abstract}
A new parameterisation of the solutions of Toda field theory is introduced. In this parameterisation, the solutions of the field equations are real, well-defined functions on space-time, which is taken to be two-dimensional Minkowski space or a cylinder. The global structure of the covariant phase space of Toda theory is examined and it is shown that it is isomorphic to the Hamiltonian phase space. The Poisson brackets of Toda theory are then calculated. Finally, using the methods developed to study the Toda theory, we extend these results to the non-Abelian Toda field theories.
\end{abstract}




\section{Introduction}

Toda theory [1-6] is a conformal field theory and provides a popular arena for the investigation of general features of two-dimensional integrable systems such as quantum groups, W-algebras and integrable hierarchies (see for example refs. [7-13] and references therein). The study of some of these properties utilises an explicit parameterisation of the solutions of the classical field equations of Toda theory and the associated Poisson bracket algebra of these parameters. Parameterisations of the solutions of the field equations of Toda theory were given in refs. [2, 3, 14]. These parameterisations describe the solutions of Toda theory on a two-dimensional Minkowski space-time. The difficulty with them is that the ranges of the associated parameters are not specified. Because of this, the solutions are not well-defined functions over all space-time (they are infinite at certain regions of space-time) and/or the solutions become complex for some choices of these parameters. For a detailed discussion of these problems in the case of Liouville theory see ref. [15]. A related problem is to find the relationship between the parameters of the solutions and the initial data of Toda theory, and define the covariant phase space and Poisson brackets of the theory. To our knowledge, these problems have not been resolved in the literature.

In a previous paper [15], we presented a new parameterisation of the solutions of the field equations of Liouville theory. In this parameterisation, the solutions of the field equations are real, well-defined functions over all of space-time. Furthermore, we showed that there is a diffeomorphism that relates the independent parameters of the solutions to the initial data of the theory, and we used this diffeomorphism to prove that the covariant and Hamiltonian phase spaces of this theory are diffeomorphic as symplectic manifolds. Finally, we proved that our parameterisation of the solutions of Liouville theory can be obtained, by using the Hamiltonian reduction methods of ref. [14], from the parameterisation of the solutions of Wess-Zumino-Witten (WZW) theory of refs. [16,17].

In this paper, we will extend these results on Liouville theory to Toda theory. 
In particular, we will show that there is a parameterisation of the solutions of the field equations of Toda theory such that these solutions are real functions over all of the two-dimensional space-time, which is taken to be either a cylinder or Minkowski space. We will also construct an explicit diffeomorphism that relates the independent parameters of the solutions to the initial data of the theory and we will use this diffeomorphism to construct a symplectic diffeomorphism between the Hamiltonian and covariant phase spaces of Toda theory. To achieve these results, we will employ the Hamiltonian reduction method of ref. [14] and the parameterisation of refs. $[16,17]$ of the solutions of the WZW model. The Poisson brackets of Toda theory are induced from those of the WZW models, and we show that these brackets are associated to the co-tangent bundle of a loop space which arises naturally in this reduction. Finally, we will describe how these results can be extended to treat the non-Abelian Toda theories [18] that can be obtained using the non-standard Hamiltonian reduction methods of refs. [19, 20].

\section{Toda Field Theory and Hamiltonian Reduction}

Let $\mathcal{L}(G)$ be a Lie algebra of a (semi)simple group $G$ and $\mathcal{H}$ be a Cartan subalgebra of $\mathcal{L}(G)$. We introduce a Chevalley basis $\left(H_{i}, E_{\alpha^{i}}, E_{-\alpha^{i}}\right)$ in $\mathcal{L}(G)$, where $\Delta \equiv\left\{\alpha^{i}, i=1, \ldots, l=\operatorname{rank} \mathcal{L}(G)\right\}$ is the space of simple roots, $H_{i} \equiv \frac{2 \alpha^{i} \cdot H}{\left|\alpha^{i}\right|^{2}}, H \in \mathcal{H}$, $E_{ \pm \alpha^{i}}$ are the step vectors of the simple roots and $\left[H_{i}, H_{j}\right]=0,\left[E_{\alpha^{i}}, E_{-\alpha^{i}}\right]=H_{i}$ and $\left[H_{i}, E_{ \pm \alpha^{j}}\right]= \pm K_{j i} E_{ \pm \alpha^{j}}$ (with no summation over $j$ ). The matrix $K \equiv\left\{K_{i j}\right\}$ is the Cartan matrix of $\mathcal{L}(G)$, i.e. $K_{i j}=\frac{2 \alpha^{i} \cdot \alpha^{j}}{\left|\alpha^{j}\right|^{2}}$. The symbols $\Phi^{+}\left(\Phi^{-}\right)$will denote the sets of positive (negative) roots, respectively, and $\Phi \equiv \Phi^{+} \cup \Phi^{-}$is the space of all roots of $\mathcal{L}(G)$. We will also use the symbols $\mathcal{L}^{+}(G)$ and $\mathcal{L}^{-}(G)$ to denote the sets of step vectors along the positive and negative roots respectively. Finally, we normalise the Killing form as follows: $\operatorname{Tr}\left(H_{i} \cdot H_{j}\right)=C_{i j}, \operatorname{Tr}\left(E_{\alpha^{i}} \cdot E_{-\alpha^{j}}\right)=\frac{2}{\left|\alpha^{i}\right|^{2}} \delta_{i j}$ and $\operatorname{Tr}\left(E_{\alpha^{i}} \cdot H_{j}\right)=0$, where $C_{i j} \equiv \frac{2}{\left|\alpha^{i}\right|^{2}} K_{i j}$. 
The Lagrangian of Toda field theory is

$$
L=-\frac{k}{8 \pi}\left(C_{i j} \partial_{+} \phi^{i} \partial_{-} \phi^{j}-\sum_{i} M^{i} \exp \left(K_{i j} \phi^{j}\right)\right)
$$

where $\phi$ is a map from a cylinder $S^{1} \times \mathbb{R}$ to $\mathbb{R}^{l}$ and $k, M^{i}$ are real, non-zero coupling constants. The pairs $(x, t): 0 \leq x<1,-\infty<t<\infty$ are the co-ordinates of $S^{1} \times \mathbb{R}$ and $x^{ \pm}=x \pm t, \partial_{ \pm}=\frac{1}{2}\left(\partial_{x} \pm \partial_{t}\right)$. The equations of motion following from the Lagrangian (1) are

$$
\partial_{+} \partial_{-} \phi^{i}+\frac{\left|\alpha^{i}\right|^{2}}{4} M^{i} \exp \left(K_{i j} \phi^{j}\right)=0
$$

In ref. [14] it was shown that Toda theory can be derived, using Hamiltonian reduction, from the WZW model with target space the group $G$, which is taken to be the maximal non-compact real Lie group associated to the Lie algebra $\mathcal{L}(G)$. An important property of this group is that it admits locally a Gauss decomposition. The field equations of the WZW model are

$$
\partial_{-}\left(\partial_{+} g g^{-1}\right)=0
$$

where $g$ is a map from a two-dimensional space-time, a cylinder $S^{1} \times \mathbb{R}$, to the group $G$ and the conserved currents (subject to field equations) are

$$
\begin{aligned}
& J_{+}(\lambda)=-\kappa \operatorname{Tr}\left(\lambda \cdot \partial_{+} g g^{-1}\right), \\
& J_{-}(\lambda)=\kappa \operatorname{Tr}\left(\lambda \cdot g^{-1} \partial_{-} g\right),
\end{aligned}
$$

where $\kappa=\frac{k}{4 \pi}, k$ is the coupling constant, and $\lambda$ is an arbitrary element of the Lie algebra $\mathcal{L}(G)$.

The WZW model reduces to Toda theory upon imposing the first class constraints

$$
\begin{gathered}
J_{+}\left(E_{\alpha^{i}}\right)=\kappa \mu^{i}, \quad \alpha^{i} \in \Delta, \quad J_{+}\left(E_{\alpha}\right)=0, \quad \alpha \in \Phi^{+}-\Delta, \\
J_{-}\left(E_{-\alpha^{i}}\right)=-\kappa \nu^{i}, \quad \alpha^{i} \in \Delta, \quad J_{-}\left(E_{-\alpha}\right)=0, \quad \alpha \in \Phi^{+}-\Delta,
\end{gathered}
$$

where the components of $\mu$ and $\nu$ are real, non-zero constants. The authors of ref. [14] solved the above constraints using the Gauss decomposition properties of the 
group $G$ and parameterised the solutions of Toda theory using the parameterisation of solutions of the WZW model of ref. [21]. This parameterisation of the solutions of Toda theory was then related to that given in ref. [2].

\section{A Parameterisation of the Toda Solutions}

To give a new parameterisation of the space of solutions of the field equations of Toda theory, eqn. (2), we will use the Hamiltonian reduction method ${ }^{\star}$ as described above and the parameterisation

$$
\begin{aligned}
g(x, t) & =U\left(x^{+}\right) \mathcal{W}\left(A ; x^{+}, x^{-}\right) V\left(x^{-}\right), \\
\mathcal{W}\left(A ; x^{+}, x^{-}\right) & =P \exp \int_{x^{-}}^{x^{+}} A(s) d s
\end{aligned}
$$

of the solutions of the WZW model (on a cylinder) given in ref. [16]. The maps $U$ and $V$ are periodic maps from the real line $\mathbb{R}$ into the group $G$, and the field $A$ in the path-ordered exponential is a $\mathcal{L}(G)$-valued periodic one-form on the real line. The expression for $g(x, t)$ in eqn. (6) is then periodic in $x$ and solves the field equations (3). The parameterisation (6) has the symmetry

$$
\begin{aligned}
& U(x) \rightarrow U(x) h(x), \quad V(x) \rightarrow h^{-1}(x) V(x), \\
& A(x) \rightarrow-h^{-1}(x) \partial_{x} h(x)+h^{-1}(x) A(x) h(x),
\end{aligned}
$$

where $h$ is an element of the loop group of $G$.

\footnotetext{
$\star$ Here we will not treat the Hamiltonian reduction method as fundamental but rather as a device to construct a parameterisation of the solutions of Toda theory. This is because of difficulties in dealing with global issues in Hamiltonian reduction. This does not affect the results of this paper.
} 
Rewriting the constraints (5) in terms of the variables $U, V, A$ of the parameterisation (3), they become

$$
\begin{gathered}
\left(\nabla_{+} U U^{-1}\right)\left(E_{\alpha^{i}}\right) \equiv\left(\partial_{+} U U^{-1}+U A\left(x^{+}\right) U^{-1}\right)\left(E_{\alpha^{i}}\right)=\kappa \mu^{i}, \\
\left(V^{-1} \nabla_{-} V\right)\left(E_{-\alpha^{i}}\right) \equiv\left(V^{-1} \partial_{-} V-V^{-1} A\left(x^{-}\right) V\right)\left(E_{-\alpha^{i}}\right)=-\kappa \nu^{i},
\end{gathered}
$$

and zero along the directions of the remaining step vectors of the negative and positive roots respectively. To solve these constraints, we recall that the group $G$ admits locally a Gauss decomposition, $G=D B C$, where the Lie algebras of the subgroups $D$ and $C$ of $G$ are $\mathcal{L}^{+}(G)$ and $\mathcal{L}^{-}(G)$ correspondingly. The subgroup $B$ of $G$ is a maximal torus of $G$ with Lie algebra $\mathcal{H}$. The parameters $U, V$ can then be decomposed as

$$
U=d_{L} b_{L} c_{L}, \quad V=d_{R} b_{R} c_{R}
$$

where

$$
\begin{aligned}
d_{L} & =\exp \left(\sum_{\alpha \in \Phi^{+}} x_{L}^{\alpha} E_{\alpha}\right), c_{L}=\exp \left(\sum_{\alpha \in \Phi^{+}} y_{L}^{\alpha} E_{-\alpha}\right), \\
b_{L} & =\exp \left(\sum_{i} \phi_{L}^{i} H_{i}\right) .
\end{aligned}
$$

and similarly for $V$. The constraints (8) can be rewritten in terms of $d_{R}, \phi_{L}, \phi_{R}$ and $c_{L}$ as follows:

$$
\begin{aligned}
& \left.\nabla_{+} c_{L} c_{L}^{-1}\right|_{\mathcal{L}^{-}(G)}=\sum_{i} \frac{\left|\alpha^{i}\right|^{2}}{2} \mu^{i} E_{-\alpha^{i}} \exp \left(K_{i j} \phi_{L}^{j}\right) \\
& \left.d_{R}^{-1} \nabla_{-} d_{R}\right|_{\mathcal{L}^{+}(G)}=\sum_{i} \frac{\left|\alpha^{i}\right|^{2}}{2} \nu^{i} E_{\alpha^{i}} \exp \left(K_{i j} \phi_{R}^{j}\right)
\end{aligned}
$$

where $\nabla$ is the covariant derivative with respect to the connection $A$ as defined in eqn. (8). 
The solutions of the Toda equations of motion can be parameterised in terms of $\phi_{L}, \phi_{R}, c_{L}, d_{R}$ and $A$. To do this, we perform a Gauss decomposition for the WZW field $g$;

$$
g=d b c
$$

where

$$
\begin{aligned}
& d=\exp \left(\sum_{\alpha \in \Phi^{+}} x^{\alpha} E_{\alpha}\right), \quad c=\exp \left(\sum_{\alpha \in \Phi^{+}} y^{\alpha} E_{-\alpha}\right), \\
& b=\exp \left(\sum_{i} \phi^{i} H_{i}\right) .
\end{aligned}
$$

The Toda field is identified as the map $\phi$ that appears in the definition of $b$ in the above equation. To project the Toda field from the expression (12) for $g$, we use the standard method of the $l$ normalised lowest weight states $\left|\lambda_{i}\right\rangle$ of finite dimensional representations of $\mathcal{L}(G)$ (with $H_{j}\left|\lambda_{i}\right\rangle=-\delta_{i j}\left|\lambda_{i}\right\rangle$ ). From eqns. (12), (6) and $<\lambda_{i}|g| \lambda_{i}>=\exp \left(-\phi^{i}\right)$, we get

$$
\exp \left(-\phi^{i}(x, t)\right)=\exp \left(-\phi_{L}^{i}\left(x^{-}\right)-\phi_{R}^{i}\left(x^{+}\right)\right)\left\langle\lambda_{i}\left|c_{L}\left(x^{+}\right) W\left(A ; x^{+}, x^{-}\right) d_{R}\left(x^{-}\right)\right| \lambda_{i}\right\rangle .
$$

It is straightforward to prove that $\phi$ in eqn. (14) satisfies the Toda equation provided that the parameters $c_{L}$ and $d_{R}$ satisfy eqns. (11) and $M^{i}=\left|\alpha^{i}\right|^{2} \mu^{i} \nu^{i}$.

To find the independent parameters of the solutions of the Toda equations, we have still to gauge-fix the symmetry (7) and solve the constraints (11). The transformation (7) can be gauge-fixed by setting $U=e$, where $e$ is the identity element of the group $G$. This implies that $\phi_{L}=0$ and $c_{L}=e$. Then the first constraint in eqn. (11) can be easily solved by taking

$$
A^{-\alpha^{i}}=\mu^{i}, \quad \alpha^{i} \in \Delta ; \quad A^{-\alpha}=0, \quad \alpha \in \Phi^{+}-\Delta
$$

where $A^{-\alpha}=\operatorname{Tr}\left(E_{\alpha} A\right), \alpha \in \Phi^{+}$. The transformations

$$
\begin{aligned}
d_{R}(x) & \rightarrow u^{-1}(x) d_{R}(x), \\
A(x) & \rightarrow-u^{-1}(x) \partial_{x} u(x)+u^{-1}(x) A(x) u(x),
\end{aligned}
$$


where $u$ is an element of the loop group of the group $D$, preserve the solution (15) and the second constraint of eqn. (11). We can use this residual symmetry to set $d_{R}=e$. In this gauge, the second constraint of eqn.(11) can be solved as

$$
A^{\alpha^{i}}=-\nu^{i} \exp \left(K_{i j} \phi_{R}^{j}\right) \quad \alpha^{i} \in \Delta ; \quad A^{\alpha}=0, \quad \alpha \in \Phi^{+}-\Delta,
$$

where $A^{\alpha}=\operatorname{Tr}\left(E_{-\alpha} A\right), \alpha \in \Phi^{+}$. To complete the discussion of the independent parameters of the solutions of the Toda equation, we observe that the constraints are independent from the parameter $c_{R}$ and so without loss of generality we can set $c_{R}=e$. This can be understood as fixing an associated symmetry in the space of parameters.

Thus the independent parameters of the solutions of Toda theory are the variables $\phi_{R}$ and the components $a^{i}$ of the connection $A$ along the directions of the Cartan subalgebra $\mathcal{H}$ of $\mathcal{L}(G)$. The solutions of the Toda theory parameterised in terms of these independent variables are

$$
\exp \left(-\phi^{i}(x, t)\right)=\exp \left(-\phi_{R}^{i}\left(x^{-}\right)\right)\left\langle\lambda_{i}\left|W\left(A ; x^{+}, x^{-}\right)\right| \lambda_{i}\right\rangle
$$

where the components of the connection $A$ corresponding to the subspaces $\mathcal{L}_{\epsilon}^{-}(G)$ and $\mathcal{L}_{\epsilon}^{+}(G)$ are given in eqns. (15), (17) and the components $a^{i}$ along the directions of the Cartan subalgebra $\mathcal{H}$ of $\mathcal{L}(G)$ are unrestricted. The above solutions $\phi$ are periodic in $x$ whenever $\phi_{R}$ and $a$ are periodic functions on the real line. The proof that the expression for $\phi$ in eqn. (18) solves the Toda field equations follows from its method of construction. Alternatively this can be shown directly. 


\section{The Phase Space Structure of Toda Theory}

The Lagrangian symplectic form $\Omega$ of a field theory is defined as the integral over a Cauchy surface of the time component of the symplectic current $S^{\mu}=$ $\delta \phi^{I} \delta\left(\partial L / \partial\left(\partial_{\mu} \phi^{I}\right)\right)$, where $\phi$ is the field and $L$ is the Lagrangian of a theory. This two-form is closed and independent of the choice of the Cauchy surface that we have used to define it. The covariant phase space $P_{C}$ of a theory is then defined as the space of parameters of the solutions of the Lagrangian equations of motion of the system, equipped with the Lagrangian symplectic form given in terms of these parameters.

The Lagrangian symplectic form of the Toda theory is

$$
\Omega=\frac{k}{16 \pi} \int_{0}^{1} d x C_{i j} \delta \phi^{i} \partial_{t} \delta \phi^{j},
$$

evaluated at $t=0$, where the map $\phi$ in eqn. (19) satisfies the Toda field equations of motion (2). We wish to insert into the form $\Omega$ the Toda solution (18), to express $\Omega$ in terms of the independent parameters of the solutions. Indeed, substituting the solution (18) into the symplectic form (19) we get

$$
\Omega=-\frac{k}{16 \pi} \int_{0}^{1} d x C_{i j}\left(\delta \phi_{R}^{i} \partial_{x} \delta \phi_{R}^{j}-2 \delta \phi_{R}^{i} \delta a^{j}\right) .
$$

where $a^{i}=-<\lambda_{i}|a| \lambda_{i}>$. The Poisson brackets are easily obtained from this, and are

$$
\begin{aligned}
\left\{\phi_{R}^{i}(x), \phi_{R}^{j}(y)\right\} & =0 \\
\left\{\phi_{R}^{i}(x), a^{j}(y)\right\} & =\frac{8 \pi}{k}\left(C^{-1}\right)^{i j} \delta(x, y), \\
\left\{a^{i}(x), a^{j}(y)\right\} & =\frac{8 \pi}{k}\left(C^{-1}\right)^{i j} \partial_{x} \delta(x, y) .
\end{aligned}
$$

Note that these brackets are the Poisson brackets on the co-tangent bundle of the loop space of $\mathbb{R}^{l}$. Finally, it can be easily seen that the Hamiltonian reduction 
described in the previous section relates the Poisson brackets of eqn. (21) to the Poisson brackets of the WZW model given in refs. [16, 17].

There are symplectic diffeomorphisms amongst the covariant phase space, the Hamiltonian phase space and the space of initial data of Toda theory. It is straightforward to prove that the latter two spaces are isomorphic. In the following we will use the parameterisation (20) of the covariant phase space of Toda theory to construct a symplectic diffeomorphism between the covariant phase space and the space of initial data of Toda theory. The space of initial data is the space of Toda fields $\phi$ and their time derivatives $\frac{\partial \phi^{i}}{\partial t}$ at $t=0$. The space of initial data can be equipped with the symplectic form induced from eqn. (19). From the Toda solution (20), we find immediately that the map between the initial data $f^{i}(x) \equiv \phi^{i}(x, 0)$, $w^{i}(x) \equiv \frac{\partial \phi^{i}}{\partial t}(x, 0)$ and our covariant phase space parameters $\phi_{R}, a^{i}$ is

$$
\begin{aligned}
f^{i}(x) & =\phi_{R}^{i}(x) \\
w^{i}(x) & =-\partial_{x} \phi_{R}^{i}(x)+2 a^{i}(x) .
\end{aligned}
$$

This map is clearly a diffeomorphism. Moreover it is a symplectic diffeomorphism from the space of initial data to the space of parameters of the solutions of the Toda theory because, as it is easy to show, it maps the symplectic form (20) into the form $\Omega=\frac{k}{16 \pi} \int_{0}^{1} d x C_{i j} \delta f^{i}(x) \delta w^{j}(x)$.

To show that the solutions of the Toda theory parameterised in terms of $a$ and $\phi_{R}^{i}$ are real, we can repeat the same arguments as those used in ref. [15] for the solutions of the Liouville theory. Indeed, for solutions analytic in the time co-ordinate $t$, this can be shown explicitly by expressing these solutions as power series in $t$ and observing that the solution $\phi$ is real at every order in the expansion. For solutions $\phi$ which are not analytic in $t$, we note that the Toda theory has a well-posed initial value problem [22], which implies that there is a unique solution of the Toda equation for each pair $(f, w)$ of initial data. Now we observe that if $\phi$ is a solution of the Toda equation and $\phi$ is complex, then its complex conjugate $\phi^{*}$ is a solution as well. However, if the initial data of $\phi$ are real, it is easy to prove 
that both $\phi$ and $\phi^{*}$ have the same initial data. Hence they must be equal $\left(\phi=\phi^{*}\right)$, and $\phi$ is real.

\section{Non-Abelian Toda Theories}

The methods developed in the previous sections can also be used to study the space of solutions of the models constructed from the WZW model with nonstandard Hamiltonian reductions. These models are the non-abelian Toda theories of refs. $[12,19,20]$. As this is a relatively straightforward generalisation of the above discussion of ordinary Toda theory, we will only sketch the main steps here. In the following, we will only consider the cases with first class constraints ${ }^{\star}$.

As in the case of Toda theory, we begin with a WZW model with target space a semi-simple group $G$ and introduce a vector $\epsilon$ on the space of fundamental coweights, i.e. the space that is dual to the space of simple roots of the Lie algebra $\mathcal{L}(G)$ of the group $G$. The vector $\epsilon$ has components that are either one or zero in the standard basis $\left\{\Lambda_{i}\right\}\left(\alpha^{j} \cdot \Lambda_{i}=\delta_{i}^{j}\right)$ of this space. Then a gradation can be introduced on $\mathcal{L}(G)$ with respect to $\epsilon$ as follows: A generator $T$ of $\mathcal{L}(G)$ has grading $n(T)$ provided that

$$
\left[H_{\epsilon}, T\right]=n(T) T
$$

where $H_{\epsilon} \equiv \epsilon \cdot H, H \in \mathcal{H}$, and $n(T)$ is an integer. In the following, we denote the grading of a generator with a superscript, i.e. $T^{n}$ means that $T$ has grading $n(T)$ in the above gradation. We can use the above gradation to decompose the Lie algebra $\mathcal{L}(G)$ into the subalgebras $\mathcal{L}_{\epsilon}^{+}(G) \equiv\{T \in \mathcal{L}(G): n(T)>0\}, \mathcal{L}_{\epsilon}^{-}(G) \equiv$ $\{T \in \mathcal{L}(G): n(T)<0\}$ and $\mathcal{L}_{\epsilon}^{0}(G) \equiv\{T \in \mathcal{L}(G): n(T)=0\}$. Notice that for the generators of the Cartan subalgebra $\mathcal{H}$ of $\mathcal{L}(G)$, the grading is zero so that $\mathcal{H} \subset \mathcal{L}_{\epsilon}^{0}(G)$; for the step vectors associated to the positive roots the grading is a positive integer $n\left(E_{\alpha}\right) \geq 0$ and for the step vectors associated to the negative roots

\footnotetext{
* Toda systems with second class constraints involve the inclusion of free fermions [23] and can be incorporated in our construction in a straightforward way.
} 
the grading is a negative integer $n\left(E_{-\alpha)} \leq 0\right.$. Next we define $P$ to be the subgroup of $G$ generated by the those generators of $\mathcal{L}(G)$ that have grading zero. It is clear that $\mathcal{L}(P) \equiv \mathcal{L}_{\epsilon}^{0}(G)$ and contains the Cartan subalgebra $\mathcal{H}$ of $\mathcal{L}(G)$. Following ref. [19], we introduce the constraints

$$
\left.J_{+}\right|_{\mathcal{L}_{\epsilon}^{-}(G)}=M,\left.\quad J_{-}\right|_{\mathcal{L}_{\epsilon}^{+}(G)}=N
$$

on the currents $J_{+}, J_{-}$of the WZW model with target space the group $G$, where $M \in \mathcal{L}_{\epsilon}^{-}(G)$ and $N \in \mathcal{L}_{\epsilon}^{+}(G)$ are constant vectors. The constant vectors $M$ and $N$ are chosen such that their components are non-zero along the directions of the subspaces of $\mathcal{L}_{\epsilon}^{-}(G)$ and $\mathcal{L}_{\epsilon}^{+}(G)$ with grading $n$ equal to -1 and +1 respectively, and their remaining components vanish.

The Lagrangian of these models is given by $L=L_{W Z W}+V_{p o t}$. where $L_{W Z W}$ is the Lagrangian of a WZW model with target space the subgroup $P$ of $G$ and $V_{\text {pot. }}$ is a potential term given by $V_{\text {pot. }}=\operatorname{Tr}\left(M p N p^{-1}\right)$ where $p$ is a map from $S^{1} \times \mathbb{R}$ into $P$. The associated field equations are

$$
\partial_{-}\left(\partial_{+} p p^{-1}\right)+\left[p N p^{-1}, M\right]=0
$$

To solve the above constraints we insert our parameterisation of the solutions of the WZW model, eqn. (6), into these constraints, and gauge-fix $U=e$ as in the case of Toda theory in section three. In this gauge, the first constraint in eqn. (24) becomes simply the requirement that the corresponding components of the connection $A$ of the WZW solution (6) be fixed to be constants, i.e.

$$
\left.A\right|_{\mathcal{L}_{\epsilon}^{-}(G)}=M .
$$

To solve the second constraint in eqn.(24), we perform a local decomposition of the group $G$ with respect to its subgroup $P, G=S P Q$, where $S$ and $Q$ are the subgroups of $G$ generated by the generators of $\mathcal{L}(G)$ with positive and negative $n$ 
grading respectively, i.e. $\mathcal{L}(S) \equiv \mathcal{L}_{\epsilon}^{+}(G)$ and $\mathcal{L}(Q) \equiv \mathcal{L}_{\epsilon}^{-}(G)^{\dagger}$. Then the parameter $V$ of the solutions of the WZW model can be decomposed as $V=s_{R} p_{R} q_{R}$ where $s_{R} \in S, p_{R} \in P$ and $q_{R} \in Q$. Next we observe that even after imposing the gauge $U=e$, the constraints (24) remain invariant under the action of the gauge transformations of eqn. (7) provided that the parameter $h$ of these gauge transformations is restricted to be an element of the loop group of the group $Q$. We can use this residual symmetry of the solution (26) to set $q_{R}$ in the decomposition of the parameter $V$ equal to the identity element of $G$, and express the solution of the second constraint of eqn. (24) in terms of the connection $A$ and $p_{R}$ as follows:

$$
\left.A\right|_{\mathcal{L}_{\epsilon}^{+}(G)}=-p_{R} N p_{R}^{-1}
$$

From eqns. (26) and (27) it is clear that the independent parameters that parameterise the solutions of the non-Abelian theories are $a$ and $p_{R}$, where $\left.a \equiv A\right|_{\mathcal{L}(P)}$ is the restriction of the connection $A$ along the directions of the Lie algebra of the subgroup $P$ of $G$, and the parameters $p_{R}$ appear in the decomposition $V=s_{R} p_{R} q_{R}$ of $V$ above.

The fields of the non-abelian Toda theory are identified with the $p$ component in the decomposition $g=s p q$ of the field $g$ of the associated WZW theory. Next, using the independent parameters $a$ and $p_{R}$, we can parameterise the most general solution of the field equations (25) of these models. Indeed from eqns (6), (26) and (27), we get

$$
p(x, t)=\left.W\left(A ; x^{+}, x^{-}\right)\right|_{P} p_{R}\left(x^{-}\right)
$$

where $\left.W\left(A ; x^{+}, x^{-}\right)\right|_{P}$ is the $P$-component of $W\left(A ; x^{+}, x^{-}\right)$in the decomposition $G=S P Q$ of the group $G$, and $A$ is the connection with components along the directions of $\mathcal{L}(S)$ and $\mathcal{L}(Q)$ given by the eqns. (26) and (27) respectively. The

\footnotetext{
$\dagger$ In the case where $\epsilon=\sum_{i} \Lambda_{i}, i=1, \ldots, \operatorname{rank} \mathcal{L}(G)$, we recover the usual Toda case, with $P=B, S=D$ and $Q=C$.
} 
components of $a,\left.a \equiv A\right|_{\mathcal{L}(P)}$, are unrestricted and together with $p_{R}$ are the independent parameters of the solutions of the theory. Finally, the periodicity of $p$ in the co-ordinate $x$ follows from the periodicity of the parameters $a$ and $p_{R}$.

The Poisson brackets of the parameters $p_{R}$ and $a$ of this theory are precisely those given by the independent parameters of a WZW model with target space the subgroup $P$ of $G$ in ref. [16]. Finally, it is straightforward to construct a map between the space of parameters of the solutions and the space of initial data of the non-abelian Toda theories, and prove that it is an isomorphism of symplectic spaces.

\section{Concluding Remarks}

The parameterisation described in section three can also be used to study Toda theory on a two-dimensional Minkowski space. Indeed, a parameterisation of the solutions of the Toda theory is given by eqn. (20) but in this case the parameters $a$ and $\phi_{L}$ are not necessarily periodic functions on the real line. A similar conclusion can be drawn for the non-Abelian Toda theories. The calculation of the symplectic form and the Poisson brackets of the theory can be formally described as in section four.

To summarise, we have presented a new parameterisation of the space of solutions of Toda theory on a cylinder. In this parameterisation, the solutions of the theory are parameterised by two sets of parameters, one of which is a set of components of a connection. This connection appeared in the expression for the solutions via its holonomy. It is worth pointing out that the holonomy of a connection entered in the parameterisation of the solutions of Liouville and WZW models $[15,16]$ as well. Thus we have found a unified and geometric understanding of the parameterisations of the solutions of the WZW, Liouville and Toda field theories. We also showed that the covariant phase space of Toda theory is isomorphic to the co-tangent bundle of the loop space of $\mathbb{R}^{l}$, with the associated Poisson brackets. In addition we constructed a diffeomorphism from the space of parameters of the 
solutions of Toda theory to the space of initial data of the theory, showed that the solutions are well-defined and real functions over all of space-time, and proved that the Hamiltonian and covariant phase spaces of Toda theory are diffeomorphic as symplectic manifolds. Finally, we discussed how these results can be extended to include the models that are obtained from the WZW model via non-standard Hamiltonian reduction methods (the non-Abelian Toda theories).

Acknowledgements: We would like to thank L. Fehér for discussions. G.P. was supported by the Commission of European Communities, and B.S. by a QEII Fellowship from the Australian Government.

\section{REFERENCES}

1. M. Toda, Phys. Rep. 18C (1975) 1.

2. A.N. Leznov and M.V. Saveliev, Lett. Math. Phys. 3 (1979) 489; Commun. Math. Phys. 74 (1980) 111.

3. P. Mansfield, Nucl. Phys. B208 (1982) 277; B222 (1983) 419.

4. D. Olive and N. Turok, Nucl. Phys. B220 (1983) 491.

5. J.-L. Gervais and A. Neveu, Nucl. Phys. B224 (1983) 329.

6. E. Braaten, T. Curtright, G. Ghandour and C. Thorn, Phys. Lett. 125B (1983) 301.

7. O. Babelon, Phys. Lett. 215B (1988) 523

8. O. Babelon, F. Toppan, and L. Bonora, Commun. Math. Phys. 140 (1991) 93.

9. J-L Gervais, "Recent Progress of The Liouville Approach to 2D Gravity and its Toda (W) Generalisations", LPTENS-92/36.

10. A. Bilal and J-L Gervais, Phys. Lett. 206B (1988) 412; Nucl. Phys. B314 (1989) 646; B318 (1989) 579. 
11. P. Mansfield and B. Spence, Nucl. Phys. B362 (1991) 294.

12. L. Fehér, L. O'Raifeartaigh, P. Ruelle, I. Tsutsui and A. Wipf, Phys. Rep. 222, No 1 (1992) 1.

13. M. Sato, RIMS Kokyuroku 439 (1981) 30; E. Date, M. Jimbo, M. Kashiwara and T. Miwa, "Transformation Groups for Soliton Equations" in Proc. of RIMS Symposium on Non-linear Integrable Systems-Classical Theory and Quantum Theory, World Scientific Publ. Co. Singapore (1983).

14. P. Forgács, A. Wipf, J. Balog, L. Fehér and L. O’Raifeartaigh, Phys. Lett. 227B (1989) 214.

15. G. Papadopoulos and B. Spence, A Covariant Canonical Formulation of Liouville Field Theory, hepth/9303076, Phys. Lett. B, in press.

16. G. Papadopoulos and B. Spence, Phys. Lett. 295B (1992) 44.

17. G. Papadopoulos and B. Spence, The Canonical Structure of the WessZumino-Witten Model, to appear in the Proceedings of the NATO ASI Conference "Low Dimensional Topology and Quantum Field Theory", Newton Institute for Mathematical Sciences, Cambridge, September 1992.

18. A.N. Leznov and M.V. Saveliev, Lett. Math. Phys. 6(1982) 505; Commun. Math. Phys. 83 (1983) 59; J. Sov. Math. 36 (1987) 699; Acta Appl. Math. (1989) 1.

19. L. O'Raifeartaigh and A. Wipf, Phys. Lett. 251B (1990) 361.

20. F.A. Bais, T. Tjin and P. van Driel, Nucl. Phys. B357 (1991) 632.

21. E. Witten, Commun. Maths. Phys. 92 (1982) 455.

22. S.W. Hawking and G. F. R. Ellis, The large scale structure of space-time, CUP (1973).

23. M. Bershadsky, Commun. Math. Phys. 139 (1991) 71. 